\title{
MRIs more accurate than mammograms but expensive
}

Two new studies that indicate MRIs are more accurate than mammograms for detecting early breast cancer contribute to a "small, but growing" body of evidence that points to MRIs as a screening tool in high-risk women, the Canadian Cancer Society says.

But the Society is reluctant to recommend that women with a family history of breast cancer or who carry the $B R C A$ genes routinely have the costly scans done.

"From our perspective, the issue is the strength of the evidence," says Heather Logan, interim director of cancer control policy. "You need to make sure that whatever screening tool you're going to use will really help to detect cancers earlier." The Society will review the studies.

A Dutch study (NEFM 2004; 351:427-37) found that screening by MRI detected $79.5 \%$ of invasive breast cancer, compared to $33.3 \%$ for mammography and $17.9 \%$ for clinical breast exam. The study screened 1909 women, including 358 who carried gene-line mutations.

A Canadian study ( $7 A M A$ 2004;292[11]:1317-25) concluded that MRIs were more sensitive and more specific in detecting small breast cancers than mammograms, ultrasounds or clinical breast exams. That study followed 236 Canadian women aged

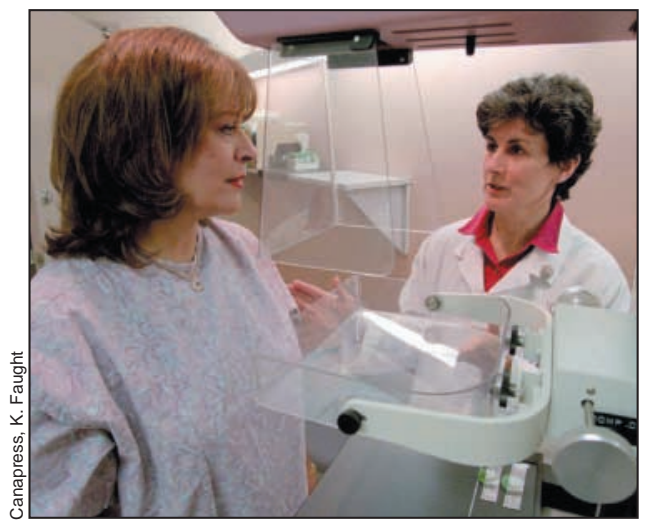

Growing evidence: Two new studies show magnetic resonance imaging is more accurate than mammography in high-risk women.
25 to 65 with $B R C A 1$ or $B R C A 2$ mutations at Toronto's Sunnybrook and Women's College Health Sciences Centre between 1997 and 2003. The women were screened through MRI, mammography, ultrasound and clinical breast exam (CBE) on the same day. Overall, the tests detected 22 cancers. MRIs detected 17 (77\%); mammography 8 (36\%); ultrasound 7 (33\%); and CBE 2 (9.1\%).

"About a third of our cancers were only detected by MRI," says Dr. Ellen Warner, a medical oncologist at Toronto Sunnybrook Regional Cancer Centre and the lead author of the study.

Sunnybrook is one of the few regional cancer centres in Canada that has an MRI available for clinical breast cancer screens and MRI-guided biopsies, so Warner is able to send women considered very high risk for scans. But Warner does not believe MRIs are appropriate for women at average risk of breast cancer.

"It's not a good strategy for average-risk women because there are so many false positives and because it's so expensive," Warner says. An MRI scan costs, on average, 4-5 times more than a mammogram, says the Canadian Association of Radiologists. But mammograms may also require further tests - adding to the cost - because they are less accurate than MRIs.

Before MRIs are used as a more general screening tool, researchers have to prove that they are better than mammography not only for detecting cancer but also for saving lives, says Warner. That will require a cohort study comparing the survival rates of women at high risk who have had MRIs with the survival rates of women who have not had MRIs.

"Everything tells us that we're probably going to be saving lives doing this, but we really have to prove it because we've been wrong before," says Warner. "Look at the hormone replacement fiasco."
Even if Canadian women at high risk of breast cancer want an MRI scan, many of them don't have access to the machines, which are in short supply in Canada. "As a general blanket statement, Canada's far behind the rest of the world in imaging equipment," says Dr. Bill Hryniuk, chair of the Cancer Advocacy Coalition of Canada.

In 2003, Canada ranked 13 out of 19 OECD countries in terms of the number of MRI scanners per million population. Canada had 147 MRIs in 2003.

Screening via MRI is a good idea, especially for women at high risk who are getting surgery on one breast, says Hryniuk.

Even for large tumours, MRIs have been shown to be more accurate than physical exams, mammography or ultrasounds in following the results of chemotherapy to shrink large breast tumours, Hryniuk says.

"Unfortunately, it's expensive, and I'm not sure that it will ever reach mass screening capability for women over the age of 50 ," he says. "I don't think doctors are using it nearly as much as they should, and that's because of a shortage of machines."

Another factor in recommending MRIs for screening is that mammography may increase the risk of cancer as well as detecting it, because of the exposure to radiation, says Hryniuk. MRIs, on the other hand, do not expose women to any radiation.

Studies of Hodgkin's survivors who underwent radiation turned up many with breast cancer, he says. "We didn't publicize it because we were afraid it would stampede women away from mammography," he says.

Although mammography has served women well, given the increased cancer risk and discomfort involved, "we'd all like to get rid of it if we could find an alternative," Hryniuk says. Laura Eggertson, CMAJ 